\title{
Generic Absorbing Transition in Coevolution Dynamics
}

\author{
Federico Vazquez, ${ }^{*}$ Víctor M. Eguíluz, and Maxi San Miguel \\ IFISC, Instituto de Física Interdisciplinar y Sistemas Complejos (CSIC-UIB), E-07122 Palma de Mallorca, Spain
}

(Received 22 October 2007; published 14 March 2008)

\begin{abstract}
We study a coevolution voter model on a complex network. A mean-field approximation reveals an absorbing transition from an active to a frozen phase at a critical value $p_{c}=\frac{\mu-2}{\mu-1}$ that only depends on the average degree $\mu$ of the network. In finite-size systems, the active and frozen phases correspond to a connected and a fragmented network, respectively. The transition can be seen as the sudden change in the trajectory of an equivalent random walk at the critical point, resulting in an approach to the final frozen state whose time scale diverges as $\tau \sim\left|p_{c}-p\right|^{-1}$ near $p_{c}$.
\end{abstract}

DOI: 10.1103/PhysRevLett.100.108702

PACS numbers: 89.75.Fb, 05.40.-a, 05.65.+b, 89.75.Hc

The dynamics of collective phenomena in a system of interacting units depends on both the topology of the network of interactions and the interaction rule among connected units. The effects of these two ingredients on the emergent phenomena in a fixed network have been extensively studied. However, in many instances, both the structure of the network and the dynamical processes on it evolve in a coupled manner [1,2]. In particular, in the dynamics of social systems (Refs. [1,3-5] and references therein), the network of interactions is not an exogenous structure, but it evolves and adapts driven by the changes in the state of the nodes that form the network. In recent models implementing this type of coevolution dynamics [2,4-12] a transition is often observed from a phase where all nodes are in the same state forming a single connected network to a phase where the network is fragmented into disconnected components, each composed by nodes in the same state [13].

In this Letter we address the question of how generic this type of transition is and the mechanism behind it. For this purpose, we introduce a minimal model of interacting binary state nodes that incorporates two basic features shared by many models displaying a fragmentation transition: (i) two or more absorbing states in a fixed connected network, and (ii) a rewiring rule that does not increase the number of links between nodes in the opposite state. The state dynamics consists of nodes copying the state of a random neighbor, while the network dynamics results from nodes dropping their links with opposite-state neighbors and creating new links with randomly selected same-state nodes. This model can be thought of as a coevolution version of the voter model [14] in which agents may select their interacting partners according to their states. It has the advantage of being analytically tractable and allows a fundamental understanding of the network fragmentation, explaining the transition numerically observed in related models [5,8-12]. The mechanism responsible for the transition is the competition between two internal time scales, happening at a critical value that controls the relative ratio of these scales.
We consider a network with $N$ nodes. Initially, each node is endowed with a state +1 or -1 with the same probability $1 / 2$, and it is randomly connected to exactly $\mu$ neighbors, forming a network called degree-regular random graph. In a single time step (see Fig. 1), a node $i$ with state $S_{i}$ and one of its neighbors $j$ with state $S_{j}$ are chosen at random, then: (1) if $S_{i}=S_{j}$ nothing happens. (2) if $S_{i} \neq$ $S_{j}$, then with probability $p, i$ detaches its link to $j$ and attaches it to a randomly chosen node $a$ such that $S_{a}=S_{i}$ and $a$ is not already connected to $i$; and with probability $1-p, i$ adopts $j$ 's state.

The rewiring probability $p$ measures the rate at which the network evolves compared to the rate at which the states of the nodes change; the extreme values correspond to a fixed network $(p=0)$, and to only rewiring $(p=1)$.

Link dynamics. - The evolution of the system can be described by the densities of two different types of links: links connecting nodes with different states or active links and links between nodes in the same state or inert links. Note that an update (either rewire or copy) only occurs when an active link is chosen.

In Fig. 1 we describe the possible changes in the global density of active links $\rho$ and their probabilities in a single time step, when a node of degree $k$ is chosen. We denote by

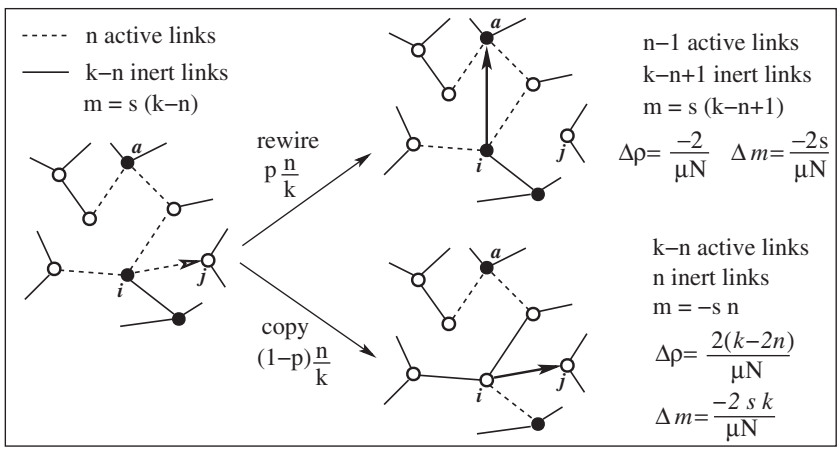

FIG. 1. Update events and the associated changes in the density of active links $\rho$ and the link magnetization $m=\rho_{++}-$ $\rho_{--}$when two neighbors $i$ and $j$ with states $S_{i}=s$ and $S_{j}=-s$ are chosen $(s= \pm 1)$. 
$n$ the number of active links connected to node $i$ before the update. With probability $n / k$ an active link $i-j$ is randomly selected. Then with probability $p$ the link $i-j$ is rewired and becomes the inert (link $i-a$ ), giving a local change of active links $\Delta n=-1$ and a global density change of $\Delta \rho=-\frac{2}{\mu N}$, where $\mu N / 2$ is the total number of links, $\mu=\langle k\rangle=\sum_{k} k P_{k}(t)$ is the number of links per node or average degree and $P_{k}(t)$ is the node degree distribution at time $t$. [Even though $P_{k}(t)$ depends on time, given that the network is constantly evolving, $\mu$ is constant because the total number of links is conserved at each time step. Furthermore, simulations show that $P_{k}(t)$ has a narrow shape with a maximum at $\mu$, as expected from the random nature of the rewiring]. With probability (1 $p$ ) node $i$ flips its state changing the state of links around it from active to inert and vice versa, and leading to $\Delta n=$ $k-2 n$ and $\Delta \rho=\frac{2(k-2 n)}{\mu N}$. Assembling these factors, the change in the average density of active links in a single time step of interval $d t=\frac{1}{N}$ is described by the master equation

$$
\begin{aligned}
\frac{d \rho}{d t} & =\sum_{k} \frac{P_{k}}{1 / N} \sum_{n=0}^{k} B_{n, k} \frac{n}{k}\left[(1-p) \frac{2(k-2 n)}{\mu N}-p \frac{2}{\mu N}\right] \\
& =\sum_{k} P_{k} \frac{2}{\mu k}\left[(1-p)\left(k\langle n\rangle_{k}-2\left\langle n^{2}\right\rangle_{k}\right)-p\langle n\rangle_{k}\right],
\end{aligned}
$$

where $B_{n, k}$ is the probability that $n$ active links are connected to a node of degree $k$, and $\langle n\rangle_{k}$ and $\left\langle n^{2}\right\rangle_{k}$ are the first and the second moments of $B_{n, k}$, respectively. In a meanfield (MF) spirit, in which the system is considered to be homogeneous, we approximate the probability that a given link is active by the average density $\rho$. Then $B_{n, k}$ becomes the binomial distribution with first and second moments $\langle n\rangle_{k}=\rho k$ and $\left\langle n^{2}\right\rangle_{k}=\rho k+\rho^{2} k(k-1)$. Replacing these expressions in Eq. (1) we obtain a closed equation for the time evolution of $\rho$

$$
\frac{d \rho}{d t}=\frac{2 \rho}{\mu}[(1-p)(\mu-1)(1-2 \rho)-1] .
$$

Equation (2) has two stationary solutions. For $p<p_{c}$, the stable solution is

$$
\rho_{s} \equiv \xi(p)=\frac{(1-p)(\mu-1)-1}{2(1-p)(\mu-1)}
$$

corresponding to an active steady state with a constant fraction of active links in the system, while for $p>p_{c}$ the stable solution $\rho_{s}=0$ corresponds to an absorbing state where all links are inert. Thus, the MF approach predicts an absorbing transition (see Fig. 2) from an active to a frozen phase at a critical value

$$
p_{c}=\frac{\mu-2}{\mu-1} \text {. }
$$

A stationary state in the active phase, characterized by a density $\xi(p)=\frac{p_{c}-p}{2(1-p)}$ of active links, is composed by links

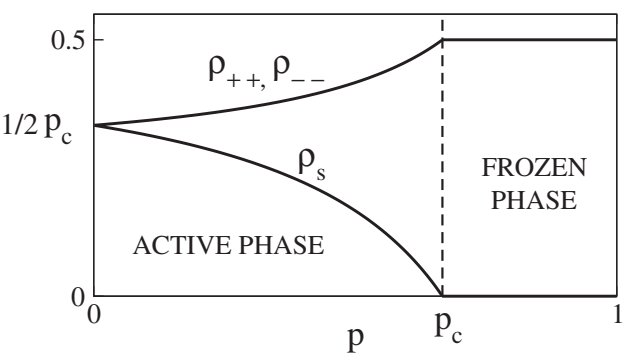

FIG. 2. Stationary density of active links $\rho_{s}$ and the two types of inert links $\rho_{++}$and $\rho_{--}$vs the rewiring probability $p$ as described by the mean-field theory for a network with average degree $\mu=4$. The critical point $p_{c}$ separates an active from a frozen phase.

continuously being rewired (evolving network) and nodes flipping their states. For $p=0$ (original voter model), the value $\xi(0)=\frac{\mu-2}{2(\mu-1)}$ agrees very well with the numerical values of the voter dynamics in different random graphs $[15,16]$. In the frozen phase, final states correspond to a fixed network where connected nodes have the same state and no more evolution is possible. The transition is continuous, with the order parameter $\rho_{s}$ changing continuously at $p_{c}$. Close and below the transition point, $\rho_{s}$ scales as $\left(p_{c}-p\right)$; thus, the MF critical exponent is 1 .

In order to obtain an insight about the structure of the network in both phases we introduce $\rho_{++}\left(\rho_{--}\right)$as the density of links connecting two nodes with states +1 $(-1)$. It can be shown that $\rho_{++}\left(\rho_{--}\right)$is related to $\rho$ and the density $\sigma_{+}\left(\sigma_{-}\right)$of $+(-)$nodes by

$$
\rho_{++}=\sigma_{+}-\rho / 2 ; \quad \rho_{--}=\sigma_{-}-\rho / 2 .
$$

Because of the conservation of the ensemble average of $\sigma_{+}$ and $\sigma_{-}$for the voter model dynamics, we have that $\sigma_{+}=$ $\sigma_{-}=1 / 2$, and therefore, $\rho_{++}=\rho_{--}=\frac{1}{2}(1-\rho)$. In the active phase the continuous rewiring of links keeps the network connected in a single component, i.e., a set of connected nodes. But, in the frozen phase only inert links are present and in the same proportion $\left(\rho_{++}=\rho_{--}=\right.$ $1 / 2$ ); thus, we expect the formation of two large disconnected components with opposite state (see Fig. 2). Therefore, the MF description reveals a fragmentation transition in the stationary structure of the network, associated with the absorbing transition at $p_{c}$.

Final states in a finite system. - The previous MF approach predicts a transition in the limit of an infinite large network. However, for any value of $p$, due to fluctuations, a finite-size network eventually reaches an absorbing state composed by inert links only. We studied the structure of the network in the final state by performing numerical simulations of the dynamics starting with a degree-regular random graph with connectivity $\mu=4$ and letting the system evolve until it was frozen. In Fig. 3(a) we plot the average size of the largest network component $S$ in the final configuration for networks with $N=250,1000$, and 4000 nodes. We observe that $S$ is very close to $N$ for values 

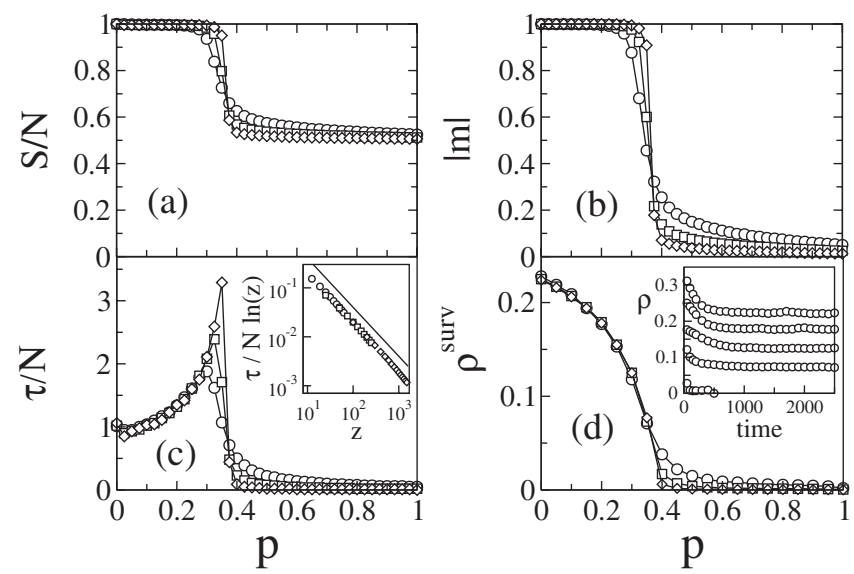

FIG. 3. (a) Average relative size of the largest network component $S$ and (b) absolute value of the link magnetization $m$ vs $p$ in the final frozen state. (c) Average convergence time $\tau$ per system size $N$ vs $p$. Inset: scaling of $\tau$ for $p \gtrsim p_{c} \simeq 0.38$, indicating that $\tau \sim \frac{N}{z} \ln (z)$ with $z=\mu\left(p-p_{c}\right) N$. The solid line has slope -1 . (d) Stationary density of active links in surviving runs $\rho^{\text {surv }}$. Inset: average time evolution of $\rho$. The averages are over $10^{4}$ realizations of networks with $\mu=4$ and sizes $N=250$ (circles), 1000 (squares), and 4000 (diamonds).

of $p$ below a transition point $p_{c} \simeq 0.38$, indicating that the network forms a single component [17]. Above $p_{c}$ the network gets disconnected into two large components and a set of components of size much smaller than $N$, giving a value of $S \simeq N / 2$.

To compare simulations with MF results, we calculated the stationary density of active links in surviving runs $\rho^{\text {surv }}$. As we show in Fig. 3(d), $\rho^{\text {surv }}$ monotonically decreases with $p$, becoming sharper with increasing system and indicating a transition from an active to a frozen phase as predicted by the MF theory. In the active phase, $\rho^{\text {surv }}$ reaches a steady value larger than zero and independent on the system size $N$, while in the frozen phase $\rho^{\text {surv }}$ vanishes in the thermodynamic limit. The critical point for the active-frozen transition $p_{c} \simeq 0.38$ calculated from Fig. 3(d) is roughly the same as for the fragmentation transition [Fig. 3(a)], suggesting that the active and frozen phases observed in infinite large systems correspond to the connected and disconnected phases, respectively, in finite systems. The MF critical point $p_{c}=2 / 3$ calculated using Eq. ([17]) with $\mu=4$ differs from the numerical value $p_{c} \simeq 0.38$ [Figs. 3(a) and 3(d)] due to correlations appearing in the rewiring process. These correlations, that are not taken into account in the analytical approximation, make the moments $\langle n\rangle_{k}$ and $\left\langle n^{2}\right\rangle_{k}$ different from the analytical values $\rho k$ and $\rho k+\rho^{2} k(k-1)$, respectively. These deviations have the overall effect of decreasing the observed critical point respect to the theoretical one.

Approach to the absorbing states. - So far, we have shown that a finite network under the coevolving dynamics experiments a fragmentation transition as the rewiring rate is increased. We now unveil the mechanism of this tran- sition by studying the evolution of the system to the frozen state.

We represent the state of the system as a point $(m, \rho)$ in the 2 dimensional space, where the coordinates are the link magnetization $m=\rho_{++}-\rho_{--}$and the density of active links, respectively. When a node of degree $k$ connected to $n$ active links is chosen, the possible changes in $m$ and $\rho$ and their respective probabilities are those described in Fig. 1. In the $(m, \rho)$ space, the system undergoes a random walk (RW) inside the triangle $0 \leq \rho+|m| \leq 1$, whose boundaries follow from the constraint relation $\rho_{--}+\rho_{++}+\rho=$ 1 . The system reaches an absorbing configuration and stops evolving when the RW hits either one of the fixed points $(-1,0)$ or $(1,0)$ (all nodes in state - or + , respectively) or a point on the fixed line $\rho=0$ (frozen mixture of - and + nodes). At the point $(-1,0)[(1,0)]$ only $--(++)$ links are present, the network is composed by a giant component, and the system is in the connected phase. Points on the line $\rho=0$ and close to point $(0,0)$ correspond to a frozen network with similar number of ++ and -- links arranged in two large + and - components (disconnected phase).

In Fig. 4 we plot trajectories of the RW in one realization for different values of $p$. For $p<p_{c} \simeq 0.38$, the motion of the RW has two stages. In the first and very short stage, the RW travels along the $m \simeq 0$ axis from the starting point ( $m \simeq 0, \rho \simeq 1 / 2$ ) to the point ( $m \simeq 0, \rho \simeq \xi_{p}$ ) that corresponds to the steady state $\rho_{s}=\xi_{p}$ in infinite large systems (see right inset of Fig. 4). In the second and long stage, the RW diffuses on the $m$ direction, corresponding to the fluctuations of $\rho$ down the steady state $\rho_{s}=\xi_{p}$, until it hits either point $m=-1$ or $m=1$ [see Fig. 3(b)]. We observe in Fig. 4 that the motion of the RW is not com-

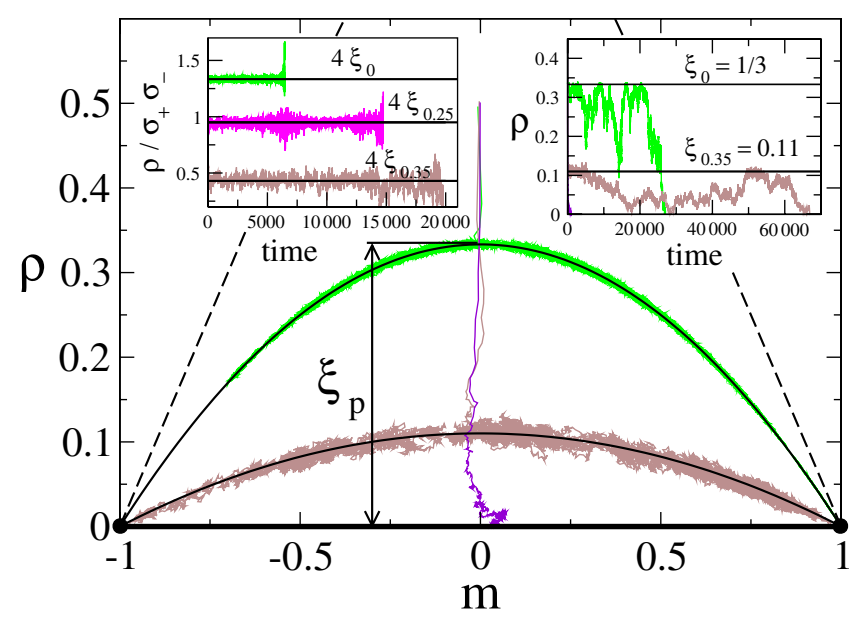

FIG. 4 (color online). Typical trajectories of the random walk for a network of size $N=10^{4}$ and $\mu=4$. The upper $(p=0)$ and lower $(p=0.35)$ parabolas are the trajectories for rewiring rates below the transition point $p \simeq 0.38$, while the quasivertical line is for $p=0.4$. Insets: Time evolution of the density of active links $\rho$ (right) and the ratio between $\rho$ and the product $\sigma_{+} \sigma_{-}$ (left) in a single realization for different values of $p$. 
pletely random but its trajectory fluctuates around a curve described by

$$
\rho_{p}(m)=\xi_{p}\left(1-m^{2}\right) .
$$

The origin of this relation is that both $\rho$ and $m$ can be expressed as functions of $\sigma_{+}$. As we observe in the left inset of Fig. 4, during one realization the ratio $\frac{\rho}{\sigma_{+} \sigma_{-}}$fluctuates around the constant value $4 \xi_{p}$. Then, using Eq. (5) we obtain $\rho=4 \xi_{p} \sigma_{+}\left(1-\sigma_{+}\right)$and $m=\rho_{++}-\rho_{--}=$ $2 \sigma_{+}-1$, from where we arrive to Eq. (6) by eliminating $\sigma_{+}$. For $p>p_{c}$, the bias to the $\rho=0$ line makes the RW hit a point close to the origin (see the $p=0.4$ trajectory in Fig. 4). Simulations show that for a fixed value $p<p_{c}$, the amplitude of the fluctuations of the RW's trajectory around its mean value $\rho_{p}(m)$ vanishes as $N$ increases. Thus, increasing $N$ has the effect of increasing the probability that the RW reaches one of the end points $m= \pm 1$ before it hits the line $\rho=0$, and therefore, most realizations end in a single component. Eventually, in the large $N$ limit, the RW has three absorbing points: either point $(-1,0)$ or $(1,0)$ (single component network) when $p<p_{c}$, and point $(0,0)$ (two components network) when $p>p_{c}$.

Convergence times. - A magnitude of interest is the average time $\tau$ to reach an absorbing state. For $p<p_{c}$, the $m$ coordinate of the walker performs a 1D symmetric random walk with an average jumping interval and its probability that scale as $1 / N$ and $\rho \sim \xi$, respectively. To reach one of the end points $m= \pm 1$ the RW needs to attempt an average of $N^{2} / \xi$ steps, and given that the time increases by $1 / N$ in each attempt, we find that $\tau \sim$ $N / \xi$. From Eq. (2) for $p \gtrsim p_{c}, \rho$ decays as $\rho(t) \sim$ $-\xi e^{4 \xi t / \mu}$. The system freezes at a time $\tau$ for which $\rho(\tau) \sim$ $1 / \mu N$, then $\tau \sim-\frac{\mu}{4 \xi} \ln (-\mu \xi N)$. Using the MF approximation $\xi(p) \sim\left(p_{c}-p\right)$ close to $p_{c}$, we obtain that $\tau \sim$ $N\left(p_{c}-p\right)^{-1} \quad$ as $\quad p \rightarrow p_{c}^{-} \quad$ and $\quad \tau \sim\left(p-p_{c}\right)^{-1} \times$ $\ln \left[\mu\left(p-p_{c}\right) N\right]$ as $p \rightarrow p_{c}^{+}$; thus, the convergence to the final state slows down at the critical point [see Fig. 3(c)].

Summary and conclusions. - In summary, the coevolution mechanism on the voter model induces a fragmentation transition that is a consequence of the competition between the copying and the rewiring dynamics. In the connected active phase, the system falls in a dynamical steady state with a finite fraction of active links. The slow and permanent rewiring of these links keeps the network evolving and connected until by a finite-size fluctuation the system reaches the fully ordered state (all nodes in the same state) and freezes in a single component. In the frozen phase, the fast rewiring dynamics quickly leads to the fragmentation of the network into two components, before the system becomes fully ordered.

The similarity between the mean-field equation for the density of active links in the coevolution voter model [Eq. (2)] and the one for the density of infected sites in the contact process [19], suggests that our model could belong to the directed percolation universality class. However, both models seem not to be equivalent given that our model possesses many absorbing states (any point on the $\rho=0$ line of Fig. 4), unlike the contact process where there is a single absorbing state characterized by the absence of infected sites. We believe that our results provide a new insight in the ongoing discussion about models with infinitely many absorbing states [20].

We thank E. V. Albano for useful comments on dynamical critical behavior, and financial support from MEC (Spain), CSIC (Spain) and EU through projects FISICOS, No. PIE200750I016, and GABA, respectively.

*federico@ifisc.uib.es

[1] T. Gross and B. Blasius, J. R. Soc. Interface 5, 259 (2008).

[2] M. G. Zimmermann, V.M. Eguíluz, and M. San Miguel, Phys. Rev. E 69, 065102(R) (2004).

[3] G. Kossinets and D. J. Watts, Science 311, 88 (2006).

[4] V. M. Eguíluz, M. G. Zimmermann, C. J. Cela-Conde, and M. San Miguel, Am. J. Sociology 110, 977 (2005).

[5] D. Centola, J.C. González-Avella, V. M. Eguíluz, and M. San Miguel, J. Conflict Resol. 51, 905 (2007).

[6] G. C. M. A. Ehrhardt, M. Marsili, and F. Vega-Redondo, Phys. Rev. E 74, 036106 (2006).

[7] I. J. Benczik, S. Z. Benczik, B. Schmittmann, and R. K. P. Zia, arXiv:0709.4042.

[8] S. Gil and D. H. Zanette, Phys. Lett. A 356, 89 (2006).

[9] P. Holme and M.E. J. Newman, Phys. Rev. E 74, 056108 (2006).

[10] F. Vazquez, J.C. Gonzalez-Avella, V. M. Eguíluz, and M. San Miguel, Phys. Rev. E 76, 046120 (2007).

[11] C. Nardini, B. Kozma, and A. Barrat, arXiv:0711.1261.

[12] B. Kozma and A. Barrat, Phys. Rev. E 77, 016102 (2008).

[13] A classical example where a social network was fragmented is described in W. W. Zachary, J. Anthro. Res. 33, 452 (1977); an extensive description on the formation of social groups can be found, for example, in S. Wasserman and K. Faust, Social Network Analysis (Cambridge University Press, Cambridge, England, 1999).

[14] R. Holley and T. Liggett, Ann. Probab. 4, 195 (1976).

[15] K. Suchecki, V. M. Eguíluz, and M. San Miguel, Phys. Rev. E 72, 036132 (2005).

[16] C. Castellano, V. Loreto, A. Barrat, F. Cecconi, and D. Parisi, Phys. Rev. E 71, 066107 (2005).

[17] We have also calculated the critical point by means of the survival probability [18]. Setting as initial condition a configuration with a few active links, the survival probability at time $t$ is calculated as the fraction of realizations that have not reached an absorbing state by time $t$. At the critical point ( $p_{c} \simeq 0.38$ ), the survival probability displays a power law distribution.

[18] M. A. Muñoz, R. Dickman, A. Vespignani, and S. Zapperi, Phys. Rev. E 59, 6175 (1999).

[19] J. Marro and R. Dickman, Nonequilibrium Phase Transitions in Lattice Models (Cambridge University Press, Cambridge, England, 1999).

[20] S.-C. Park and H. Park, Phys. Rev. E 76, 051123 (2007). 